\title{
Improvement of the Effectiveness of Treatment Patients with Tuberculosis Spondylitis
}

\section{GG Holka*, VV Vesnin, VV Burlaka, AO Oliinyk and OG Fadieiev}

Kharkiv National Medical University, Kharkiv, Ukraine

*Corresponding Author: GG Holka, Kharkiv National Medical University, Kharkiv, Ukraine.
Received: April 01, 2021

Published: May 11, 2021

(C) All rights are reserved by GG Holka., et al.

\begin{abstract}
The protocols of clinical and radiological examination, analysis of treatment of 60 patients with active tuberculous spondylitis have become the clinical material of this study.

The main group included patients $(n=30)$ with TS of the thoracic and lumbar vertebrae operated with the use of a sliding titanium cage for ventral intercostal spondylodesis.

The comparison group includes patients $(n=30)$ using traditional approaches to the surgical treatment of the TS.

The comparative study of the effectiveness of treatment of patients of the main and control group with TS showed that the use of the proposed technique in patients of the main group made it possible to significantly improve the results of treatment.

Tuberculosis spondylitis (TS) in the structure of bone-joint tuberculosis in adults occupies a leading position and reaches 40 - $61.5 \%$ and it presents the great medical and social problem $[2,4,8]$.
\end{abstract}

Keywords: Tuberculosis Spondylitis (TS); Lumbar Vertebrae; Spondylodesis

\section{Introduction}

The modern approach in the treatment of tuberculosis spondylitis is based on the use of radical, radical and reconstructive operations, the use of which allowed in $70.5 \%-80 \%$ of cases to achieve good treatment results, which significantly exceeds the effectiveness of conservative treatments. But to date, the issue of adequate stabilization of the spine remains relevant. Anterior spondylosis as its fundamental component in the vast majority of cases is performed by autotransplant $[4,6,9]$.

Despite the good result of anterior bone plasty in the near postoperative period, in the separated - the frequency of adverse effects is increasing from 16 to $40 \%[5,6,8]$.

Inpatient treatment of a patient with active spinal tuberculosis at present continues for at least a year and the complex of surgical interventions does not always give the desired result. Prolonged hypodynamia leads to the appearance of pronounced dystrophic changes in the musculoskeletal system and internal organs, which complicates the rehabilitation of patients in the postoperative period in specialized hospitals or sanatoriums.

Until now, the issue of approaches to preoperative treatment remains unresolved (the expediency of using the second-row $\mathrm{ABP}$, the timing of pre-peripheral treatment, the use of existing achievements of vertebrology in the conditions of $\mathrm{ft}$ and orthopedia (modern methods of anterior spine and lodes).

\section{Purpose of the Study}

Increasing the effectiveness of treatment of patients with tuberculosis spondylitis, by improving the etiological diagnosis and surgical treatment.

\section{Materials and Methods of Research}

The clinical material of this study was the protocols of clinical and X-ray examination, analysis of treatment of 60 patients with active tuberculosis spondylitis, operated on the basis of the depart- 
ment of bone-joint tuberculosis OPTD No 1 in Kharkiv according to the contract No 1031/04-16 of April 08, 2016 and the clinical base of the Department of Traumatology and Orthopedics of KhNMU in the departments of traumatology and orthopedics of the Non-profit Municipal Enterprise "City Clinical Hospital of Emergency and Emergency Medical Care named after. Prof. O.I. Meshaninova" of Kharkiv City Council in the period from 2012 to 2017, where patients with TS were treated, did not pose any threat to others in epidemiological terms, the so-called "closed" forms of the disease (without the presence of burrows, connective pulmonary tuberculosis). Patients are divided into two groups, depending on the approaches to preoperative training and the method of surgical stabilization of the destructive specific spine process (main group and control).

Patients of the control and main group underwent preoperative training, radical decompressive - stabilizing operations of necrosectomy, decompression of the neuro-vascular structures of the vertebral canal with subsequent interthyl spondylodesis were performed.

The main group (Gr. No. 1) includes patients $(n=30)$ with tuberculosis spondylitis of the thoracic and lumbar vertebrae, operated with the use of a solvent titanium eight for ventralinterthyl spondylodesis. The peculiarity of the preoperative preparation of the pack is the nts of this group was the mandatory conduct of a bacteriological study before the start of treatment with the determination of the sensitivity of MBT to ABP, the appointment of a short-term intensive course of specific antibacterial therapy, taking into account the results of the study within 2 - 3 weeks with the subsequent surgery. The basis for such a short-term preoperative preparation was the results of experimental research [1]. The peculiarity of the surgical stage of treatment in this group of patients was the performance after the sanitizing stage (decompression necrectomy) of the anistonium and lodez using atelescopic titanium cage.
The comparison group (group No. 2) includes patients $(n=30)$ with tuberculosis spondylitis of the thoracic and lumbar vertebrae using traditional approaches to surgical treatment of TS - preoperative preparation using 3-5 specific ABPs without determining sensitivity for 2 - 3 months followed by surgery - decompressive necrotomy of the affected vertebrae and anterior spondylosis autotransplant taken from a fragment of the rib (in the case of thoracic access) or from the wing of the lumbar bone during surgery on the lumbar spine. This group of patients was analyzed retrospectively (based on the analysis of patient's medical histories, who were undergoing treatment in the KST department of Kharkiv OTL No1 in 2002 - 2005.

\section{Criteria for including patients in the study:}

- $\quad \mathrm{TC}$ in the active phase (verified pathomorphologically and/or bacteriologically.

Criteria for excluding patients from the study:

- $\quad$ Patients over 75 years old;

- $\quad$ Previously operated patients on the spine;

- Patient and with the presence of decompensated concomitant (not tuberculosis) impressions.

By age, sex, number of affected vertebrate motor segments, activity of infectious inflammatory process, patients of the main group and comparison group are almost identical, therefore, comparing the results of treatment of patients of both groups in the nearest ( 3 months) and remote periods ( $1-2$ years) is considered quite correct.

In the main group and comparison group among male patients, $66.4 \%$ and $59.94 \%(n=20 ; n=18)$, women respectively $33.4 \%$ and $40.06 \%(\mathrm{n}=10 ; \mathrm{p}=12)$ (Table 1).

\begin{tabular}{|c|c|c|c|c|c|c|c|c|c|c|c|c|c|c|c|}
\hline & & \multicolumn{10}{|c|}{ Age, years } & \multicolumn{4}{|c|}{ Just } \\
\hline & \multicolumn{2}{|c|}{$21-30$} & \multicolumn{2}{|c|}{$31-40$} & \multicolumn{2}{|c|}{$41-50$} & \multicolumn{2}{|c|}{$51-60$} & \multicolumn{2}{|c|}{$>60$} & \multicolumn{2}{|c|}{ Abs } & \multicolumn{2}{|c|}{$\%$} & \multirow[b]{2}{*}{ Ii } \\
\hline \multicolumn{2}{|c|}{$\begin{array}{l}\text { Patient } \\
\text { groups }\end{array}$} & And & Ii & And & Ii & And & Ii & And & Ii & And & Ii & And & Ii & And & \\
\hline & Abs. & 1 & 2 & 5 & 4 & 12 & 11 & 9 & 10 & 3 & 3 & 30 & 30 & & \\
\hline Just & $\%$ & 3,33 & 6,66 & 16,65 & 13,32 & 39,96 & 36,63 & 29,97 & 33,3 & 9,99 & 9,99 & & & 100 & 100 \\
\hline
\end{tabular}

Table 1: Distribution of patients by age. 
The average age of patients in the main group and comparison group was $38.2 \pm 9.6$ and $40 \pm 10.8$ years, respectively ( $p>0.05$ ).

When comparing age indicators in both groups, the following feature was found. It was established that young patients aged 20 to 30 are significantly less over sutured by the incidence of TS than individuals over 40 years of age.

Among the patients, individuals with damage to the bodies of the vertebrae prevailed. Only 2 patients of the first group had affected the hind parts of the vertebrae: transverse and articular processions, arcs.
The distribution of patients by localization of the lesion and the number of affected vertebrae are given in table 2 . In the overwhelming number of observations in both groups, there were damage to the bodies of two vertebrae: in group I - 25 (83.25\%), in the second group - also 25 (83.25\%) patients. Most often, both in the first and in the second groups, the lump lumbar spine was affected. It should be noted that when localizing the destructive specific process in these spine regions, the involvement of three vertebrae bodies in the inflammatory process was noted more often.

For a qualitative assessment of neurological disorders, we used the Frankel/Asia scale [7].

\begin{tabular}{|c|c|c|c|c|c|c|c|c|c|c|c|c|}
\hline \multirow[t]{4}{*}{ Localization } & \multicolumn{8}{|c|}{ Number of affected vertebrae } & \multirow{2}{*}{\multicolumn{4}{|c|}{ Just }} \\
\hline & \multicolumn{4}{|c|}{2} & \multicolumn{4}{|c|}{3} & & & & \\
\hline & \multicolumn{2}{|c|}{ And gr. } & \multicolumn{2}{|c|}{$2^{\text {nd }}$ city } & \multicolumn{2}{|c|}{ And gr. } & \multicolumn{2}{|c|}{$2^{\text {nd }}$ city } & \multicolumn{2}{|c|}{ And gr. } & \multicolumn{2}{|c|}{$2^{\text {nd }}$ city } \\
\hline & Abs & $\%$ & Abs & $\%$ & Abs & $\%$ & Abs & $\%$ & Abs & $\%$ & Abs & $\%$ \\
\hline Pectoral & 7 & 23,31 & 8 & 26,64 & 3 & 9,99 & 2 & 6,66 & 10 & 33,3 & 10 & 33,3 \\
\hline Breast Lumbar & 8 & 26,64 & 9 & 29,97 & 2 & 6,66 & 3 & 6.66 & 10 & 33,3 & 12 & 39,96 \\
\hline Across the Viy & 6 & 19,98 & 5 & 16,65 & - & 0 & - & 0 & 6 & 19,98 & 5 & 16,65 \\
\hline Lumbar sacruff & 4 & 13,32 & 3 & 9,99 & - & 0 & - & 0 & 4 & 13,32 & 3 & 9,99 \\
\hline Just & 25 & 83,25 & 25 & 83,25 & 5 & 16,65 & 5 & 16.65 & 30 & 100 & 30 & 100 \\
\hline
\end{tabular}

Table 2: Distribution of patients by localization and number of affected vertebrae.

It should be noted that patients of both groups did not differ in the degree of neurological disorders.

All patients with the first diagnosed TC before the start of treatment were carried out a comprehensive examination (clinical and X-ray, laboratory, instrumental), which allowed to clarify the precommunicability and activity of a specific process, to identify complications and concomitant diseases, violations of the functions of various organs and systems.

In patients of the main group, a puncture etiological diagnosis of the disease was carried out without fail before the start of treatment. Such a survey allowed to determine the localization, size, propagation and nature of the destructiveness of the bodies of the vertebrae, as well as the condition of paravertebral tissues adjacent to the cell (the presence of abscesses, abscess-like shadows).

For the treatment of patients with TC on the basis of traditional approaches (control group of 30 patients, we used methods of sur- gical treatment, including rehabilitation of abscissions, resection or necrotomy of the focus of destructiveness, stabilization of the affected spine sections with the help of an anistonior or autotransplant and immobilization (unloading) in the pre- and postoperative period with the use of bedding.

Operations were carried out in the amount of neurectomy, radical resection of the foci of destructive disease, anterior side spondylosis with auto transplants. It should be noted that the long pre- and postoperative bedding regimen in patients of this group, necessary for long-term ( 2 - 3 months) preoperative training and due to the lack of stable fixation of the operated segment of the spine, which also required the observance of bedding within the next 2 - 4 months. especially during the transition to a more active orthopedic regimen (verticalization and walking with weeds). Functional changes in the cardiovascular system in the control group were detected in 20 patients (66.6\%), in the form of lymphostasis in the lower extremities in 9 patients (29.99\%). In the 
early postoperative period, symptoms of venous insufficiency of the lower extremities were noted.

It is important to note that surgery for patients of both groups was carried out as a stage of treatment, after basic antibacterial and pathogenetic therapy.

The fundamental differences in the treatment of patients of the main and control groups were as follows:

1. Mandatory study of pathological material of the focus of destructiveness in patients of the first group with determination of CBT sensitivity to ABP.

2. Short-term intensive ABT course for 2 - 3 weeks, taking into account the sensitivity of MBT.

3. When performing surgery in patients of the main group, a swout telescopic titanium cage was used instead of an autotransplant.

During surgery on the thoracic and lumbar spine, preference was given to the front access, which are described in detail in the monograph of O.O. Korzh, R.R. Lyashynsky, M.I. Khvysyuk [3].

In the patients of the control group, typical resections of two adjacent affected vertebrae were used, kyphotic deformity was decreased, and autotransplants were wedged into the formed groove.

In all cases, the kyphotic deformation of the vertebrae, if possible, was eliminated by resection, wedgeing the deformation zone and completely substitution of the resulting defect with autotransplants.

The next stage of the operation was actions aimed at eliminating spinal disorders. Elimination of soft compression (pus, caseous masses, granulations, scars) or hard brain compression by bone sequestration or deformation angle by resection of this angle and reconstruction of the spinal canal.

Below is a clinical example of surgical treatment of TS in the thoracic region and spine using autotransplants in patients of the control group (Figure 1).

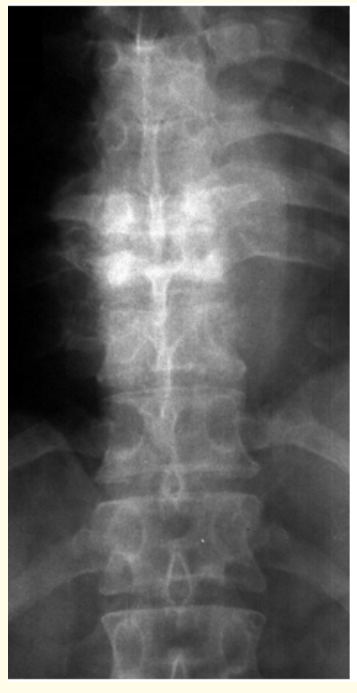

a)

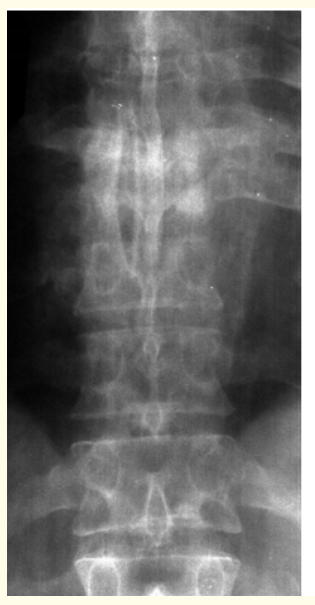

c)

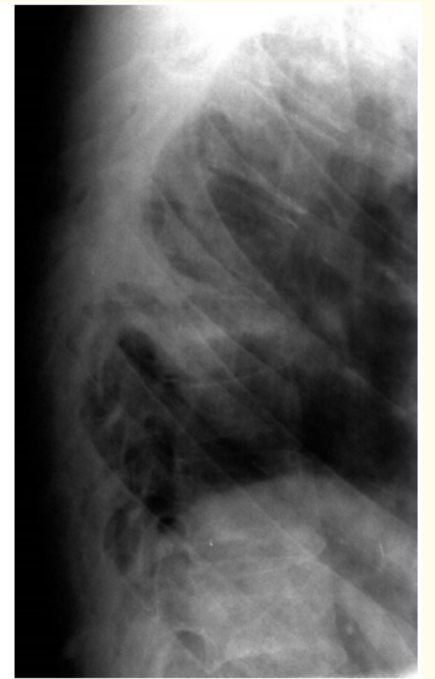

b)

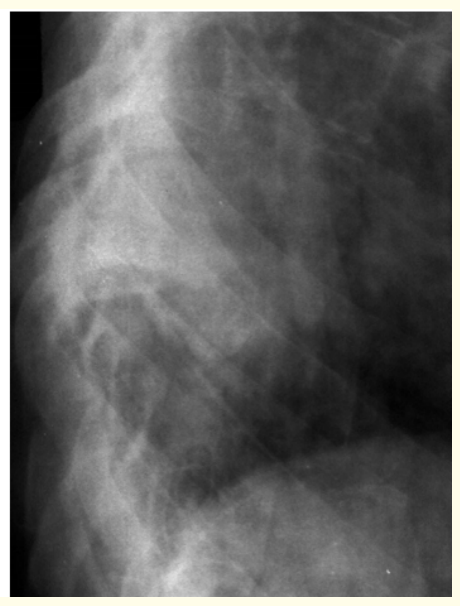

d)
Figure 1: Photoprinting of X-rays of the patient $K$., medical history No. 5280: $a$, b) before the operation (contact destruction is visualized in the segment D7-D8 with the presence of influting abscess, kyphotic deformity in the destruction zone); $c$, d) after the operation (decompressive necrotomy of the affected segment of the spine with wedging spondylosis autotransplant from the rib, which was resisted during the performance of anterior access). 
After performing the sanitizing stage of surgery, patients of both groups performed anterior spondylosis using autotransplant (control group), or exodus titanium cage - the main group.

In our opinion, the use of cages for pre-spondylosis in patients with TS compared to autotransplants has a number of advantages:

It is known that titan implants for front access in terms of their biomechanical parameters surpass the bone. Such structures are able to withstand a load of more than 3 tons. Metal fatigue test showed that cages are able to withstand 5 million cycles with a load of one ton $[2,5,8,9]$.

Along with this, telescopic body-containing implants allow you to provide:

- Restoration of the height of the inter-man space.

- Fixation and stabilization of the support columns of the spine.

- Individually select the final parts in accordance with the anatomy of the patient.

- Adjust the sagittal deformity of the spine due to the possibility of dissent.

Below are clinical examples of surgical treatment of TS in the thoracic and lumbar spine using a fork telescopic cage - the main group (Figure 2).

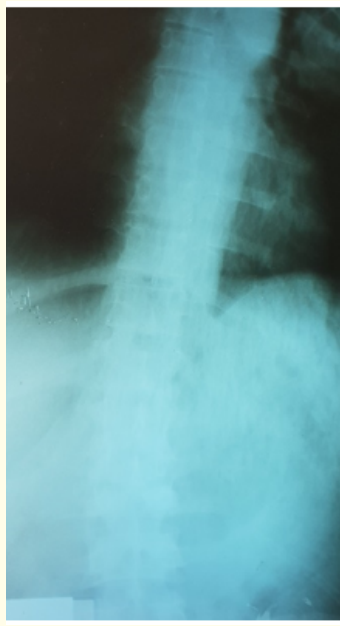

a)

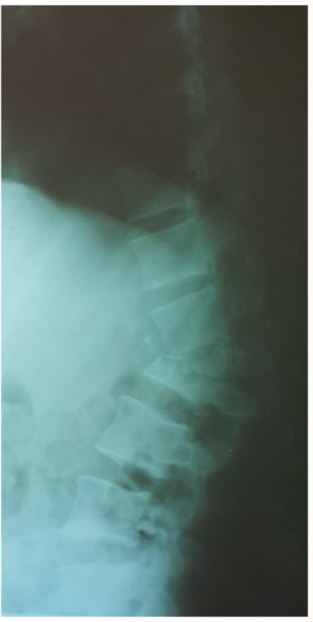

b)

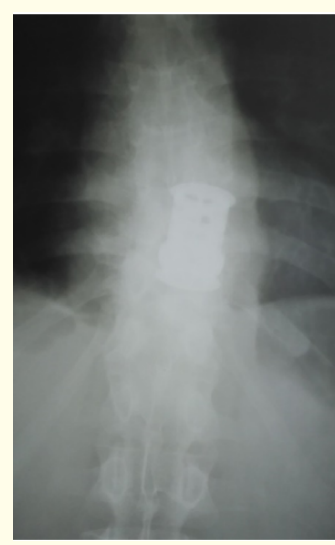

c)

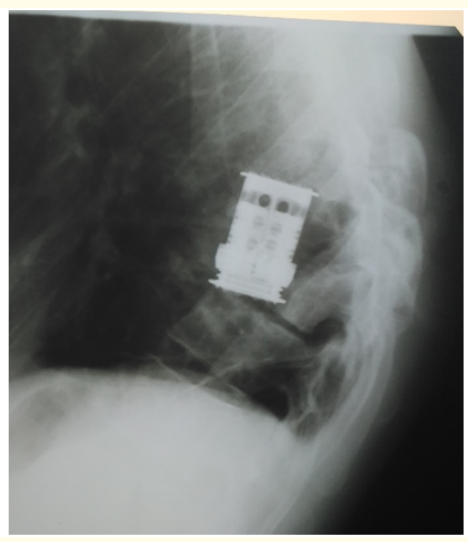

d)
Figure 2: Photoprints of X-rays of the patient K., medical history No. 5280: $a$, b) before the operation (contact destruction is visualized in the segment D10-D11 with the presence of influting abscess, kyphotic deformity in the destruction zone); c, d) after the operation (decompressive necrotomy of the affected segment of the spine with the wedging spondylosis titanium telescopic cade).

\section{Results of Research and Discussion}

We evaluated the long-term results of surgical treatment of spondylitis, based on anatomical and functional results, the presence of neurological symptoms and the degree of recovery of the patient. Data from the literature confirm the similarity of the views of other authors on the criteria for assessing the surgical treatment of spondylitis.

To simplify the analysis of treatment results, we divided the results into good, satisfactory and unsatisfactory (used the Ulrich, Mushkin scale) [7].

Among the good ones was the restoration of sufficient musculoskeletal function of the spine in the presence of radiologically verified bone block between the resected vertebrae, normalization of the hemogram and the absence of patient complaints.

Satisfactory results include, when in the presence of a bone block, minor pain and limitation of musculoskeletal function of the spine persist.

Unsatisfactory include the results of treatment while maintaining restriction of movement and emptiness of the spine, pronounced degenerative changes, the absence of bone block. 
Remote results of TS treatment were studied by us in 26 patients of group I (86.7\%) and 25 patients of group 2 (83.3\%) from 1 to 10 years. The remaining patients were residents of remote regions of Ukraine and they could not trace the remote results of treatment. Clinical, X-ray, neurological examination, as well as in the cases indicated, computed tomography did not detect recurrences of the disease. At the same time, excellent results were obtained in 14 patients of the main group (54\%) and 8 - control group (32\%); good - in $71(24.7 \%)$ patients of the main group and in 7 (28\%) patients of the control group; satisfactory - in $4(15.4 \%)$ patients of the main group and in 7 (28\%) control group, unsatisfactory - in 3 (12\%) patients of the control group, there were no unsatisfactory results in patients of the main group.

In patients with severe neurological complications of TC, only in 2 cases no positive effect was obtained. Of the 7 patients with the initial picture of the lower paraplegia (types $\mathrm{A}$ and $\mathrm{C}$ according to Frankel) as a result of treatment, 5 have restored the capacity for independent movement, including 3 - functionally complete gait (types E and D according to Frankel).

Thus, the generally accepted methods of preoperative management of patients, operations and postoperative treatment, involving a long bed rest, lead to a slowdown in the regeneration process in the area of resection of the focus of destruction in the vertebral segments. As a result, osteoporosis of bone tissue develops, and the formation of bone ankylosis of resected vertebrae is significantly slowed down.

Delayed formation of bone blocks, muscle atrophy due to prolonged hypodynamics, functional disorders of some organs and systems of the body do not allow for early rehabilitation of patients, as a result of which they are forced to use corsets and crutches for up to 2 - 3 years. All this period they are disabled 1 - 2 groups.

Analysis of the traditional approach to the treatment of spondylitis on the basis of analysis of treatment results of the control group of patients allowed to conclude that preoperative and postoperative periods on the background of strict bed rest, the total duration of which was depending on the localization of the process from 2 - 3 to 4 - 6 months. hypodynamia, which negatively affected the functional state of organs and systems, significantly slowed down reparative processes in bone tissue. Thus, the formation of callus between resected vertebral bodies occurred in the period from 1 to 2 years. The output of patients with disabilities of the $1^{\text {st }}$ and $2^{\text {nd }}$ groups was about $90 \%$.

Moreover, out of 30 patients of the main group, only 5 people changed jobs, 7 received group III disability, 18 patients had group II disability, and the others returned to their previous work after 6 - 8 months.

It should also be noted that the average duration of inpatient treatment in patients of the main group was significantly shorter than in the control group (main group $96 \pm 12$ days), control (190 \pm 21 ).

In our opinion, such a significant difference in terms of inpatient treatment is due primarily to the etiological diagnosis to identify the sensitivity of the Office to ABP, followed by the appointment of ATB, taking into account the study, short-term intensive preoperative preparation and use of surgical interventions for modern interventions, which allows much earlier rehabilitation and verticalization of operated patients, significantly reducing inpatient treatment.

Criteria for comparing the results of treatment of patients in the main and control groups were also: clinical, radiological, physiological. Naturally, the most important in the study were clinical criteria, such as: the effectiveness of pain relief, the time after surgery, as well as the possibility of activation of patients after surgery. The timing of the inpatient treatment and rehabilitation.

Clinical outcomes of treatment of patients of the main and control groups in the near and distant periods differed significantly.

Upon admission to the hospital, all patients of the main and control groups of the comparison complained of pain in the affected spine, which was exacerbated with minimal movement. Examination and palpation determined the tension of the long muscles of the back and the forced position of the patients ("root symptom" according to Kornev). In addition, all patients had severe pain on palpation in the paravertebral areas of the affected segments. Violation of the shape of the spine in the sagittal plane, as a rule, was significant and consisted of a local increase in thoracic kyphosis (with lesions of the thoracic vertebrae) or correction of lumbar lordosis (with inflammation in the lumbar vertebrae). 
Clinical characteristics of patients with tuberculous spondylitis from the main and control groups at the initial examination are presented in table 3 .

\begin{tabular}{|c|c|c|c|c|}
\hline \multirow[t]{2}{*}{ Tags/Groups } & \multicolumn{2}{|c|}{$\begin{array}{l}\text { Basic }(n= \\
30 ; 100 \%)\end{array}$} & \multicolumn{2}{|c|}{$\begin{array}{c}\text { Control } \\
(n=30 ; \\
100 \%)\end{array}$} \\
\hline & abs. & $\%$ & abs. & $\%$ \\
\hline $\begin{array}{l}\text { Angular kyphosis at the level of } \\
\text { the affected segments }\end{array}$ & 26 & 86,7 & 24 & 80,0 \\
\hline Paravertebral muscle hypertonia & 30 & 100,0 & 30 & 100,0 \\
\hline $\begin{array}{l}\text { Limited movements in the affected } \\
\text { spine }\end{array}$ & 30 & 100,0 & 30 & 100,0 \\
\hline Lower paraparesis & 15 & 50,0 & 16 & 53,3 \\
\hline Lower paraplegia & 9 & 30,0 & 8 & 26,7 \\
\hline Sphincter disorders & 10 & 30.0 & 11 & 36,7 \\
\hline Inflowing abscesses & 24 & 80,0 & 23 & 76,7 \\
\hline Fistulas & 5 & 16,7 & 6 & 20,0 \\
\hline $\begin{array}{l}\text { Pain intensity according to VAS, } \\
\qquad \mathrm{mm}(\mathrm{M} \pm \mathrm{m})\end{array}$ & \multicolumn{2}{|c|}{$61,5 \pm 7,8$} & \multicolumn{2}{|c|}{$64,0 \pm 7,6$} \\
\hline
\end{tabular}

Table 3: Clinical characteristics of patients with tuberculous spondylitis from the main and control groups at the initial examination.

At follow-up a year later, patients in the control group complained of pain in the area of specific vertebral impression after exercise. At rest, recurrent pain in patients of the main group was observed in only 3 (9.99\%) patients, and in patients of the comparison group in 10 (33.3\%) cases. On examination of patients in the main group, the tone of the paravertebral muscles of the back is preserved, on palpation of the paravertebral areas of adjacent segments, the pain syndrome was not reproduced. While in 10 $(33.3 \%)$ patients of the comparison group, after the peak after surgery, they complained of constant pain in the operated spine at rest with increased pain after minimal loads, especially in the paravertebral areas of the affected vertebrae. Examination of patients in the comparison group revealed malnutrition in the paravertebral areas in 16 (53.28\%) patients, and on palpation of tense muscles and pain in the paravertebral areas in 20 (66.6\% of cases). The main cause of pain and malnutrition in paraverterbral areas in patients of the comparison group were postoperative ischemic changes in the spinal musculoskeletal complex with signs of structural and functional insufficiency, the main cause of which, in our opinion, was prolonged bed rest.

In the comparative analysis of the nearest and separate results of surgical treatment of patients of both groups with TC of thoracic and lumbar vertebrae the results which brightly testified to high clinical efficiency of the offered technique of treatment of patients of the main group are received. The main supporting facts of improving the effectiveness of treatment of patients in the main group in the study were to reduce the stage of preparation for radical surgery, reduce the number of inflammatory complications, significantly reduce the duration of inpatient treatment, improve quality of life.

Clinical characteristics of patients with tuberculous spondylitis from the main and control groups after surgery are presented in table 4.

\begin{tabular}{|c|c|c|c|c|}
\hline \multirow[t]{2}{*}{ Tags/Groups } & \multicolumn{2}{|c|}{$\begin{array}{c}\text { Basic }(n=30 ; \\
100 \%)\end{array}$} & \multicolumn{2}{|c|}{$\begin{array}{c}\text { Control }(n=30 ; \\
100 \%)\end{array}$} \\
\hline & abs. & $\%$ & abs. & $\%$ \\
\hline $\begin{array}{c}\text { Paravertebral muscle } \\
\text { hypertonia }\end{array}$ & 2 & 6,7 & 16 & 53,3 \\
\hline Lower paraparesis & 4 & 13,3 & 8 & 26,7 \\
\hline Lower paraplegia & - & - & 3 & 10,0 \\
\hline \multirow[t]{2}{*}{ Sphincter disorders } & 2 & 6,7 & 5 & 16,7 \\
\hline & \multicolumn{4}{|c|}{$(\mathrm{M} \pm \mathrm{m})$} \\
\hline $\begin{array}{c}\text { Pain intensity according } \\
\text { to VAS, mm }\end{array}$ & \multicolumn{2}{|c|}{$22,2 \pm 7,8$} & \multicolumn{2}{|c|}{$34,9 \pm 9,1$} \\
\hline $\begin{array}{l}\text { Term of hospital stay, } \\
\text { bed-days }\end{array}$ & \multicolumn{2}{|c|}{$96 \pm 12$} & \multicolumn{2}{|c|}{$190 \pm 21$} \\
\hline
\end{tabular}

Table 4: Clinical characteristics of patients with tuberculous spondylitis from the main and control groups after surgery.

Thus, the analysis of remote results carried out in the examined patients of the main group with intensive short-term (2 - 3 weeks) antibacterial therapy (taking into account the sensitivity of MBT to $\mathrm{ABP}$ ) with the subsequent implementation of surgery using a telescopic titanium fork cage, showed that this technique allows to achieve high treatment efficiency. 
Analysis of remote results indicates significantly better results of treatment of patients of the main group.

Further increase in the effectiveness of treatment of patients with TC in our opinion will depend primarily on the timeliness of diagnosis of this disease, the study of blood supply to the spinal cord, the development of schemes of directed neurotrophic therapy, improvement of neurostimulation treatment methods.

The frequency of complications observed in the postoperative period in patients of both groups was also studied. 2) postoperative pneumonia, exudative pleurisy; 3) exacerbation of the inflammatory process with the formation of minks; 4) nephrosis of the skin edges of the wound and its secondary nougat. Progression of deformation in the area of specific spine impression. These complications were detected after 4 - 6 months and in a longer period after surgery. Types of complications and their frequency are presented in table 5.

\begin{tabular}{|c|c|c|}
\hline Complications & $\begin{array}{c}\text { Main Group } \\
\text { No.30 }\end{array}$ & $\begin{array}{c}\text { Control group } \\
\text { No.30 }\end{array}$ \\
\hline Common complications & & 1 \\
\hline Postoperative pneumonia & 1 & 1 \\
Exudative pleurisy & - & 2 \\
Local inflammatory (serous & 2 & 2 \\
inflammatory) & & 1 \\
\hline Progression of the disease & 0 & 3 \\
Nigno-who is the one who can & 1 & \\
look & 0 & \\
Fractures, displacement of & & \\
autotransplant, cage & & \\
\hline
\end{tabular}

Table 5: Structure of complications in the nearest and distant postoperative periods in patients of the main and control group.

It should be noted that out of 30 patients of the main group, only 5 people changed jobs, 7 received a group III disability, 18 patients received group II disability, the rest returned to their previous work after 6 - 8 months.

It is important to emphasize that the average period of inpatient treatment in patients of the main group was significantly less than in the control group (main group $96 \pm 12$ days), control (190 \pm 21 ).
In our opinion, such a significant difference in the timing of inpatient treatment is primarily due to the conduct of etiological diagnostics with the detection of mbt sensitivity to ABP, with the subsequent appointment of ABT, taking into account the study carried out, conducting short-term intensive preoperative training and using modern structures for anterior spondylosis during surgery, which allows much earlier rehabilitation and verticalization of operated patients, significantly reducing inpatient treatment.

\section{Conclusion}

1. The results of the experimental study substantiate the effectiveness of short-term preoperative ABT for 3 - 4 weeks, followed by surgery without the risk of generalization of the disease.

2. The use of the proposed methods of etiological diagnosis can significantly accelerate the correct formulation of clinical diagnosis and the appointment of adequate ABT.

3. The comparative study of the effectiveness of treatment of patients of the main and control group with TS showed that the use of the proposed technique in patients of the main group made it possible to significantly improve the results of treatment by significantly reducing the stage of preparation for radical operations, reducing the number of complications, reducing the time of inpatient treatment and improving the quality of life.

\section{Conflict of Interest}

The authors of the publication declare that there is no conflict of interest.

\section{Bibliography}

1. Holka GG., et al. "Experimental modeling of tuberculosis spondylite”. Injury 34 (2018): 68-75.

2. Diagnosis and treatment of extrapulmonia tuberculosis. Practical Guide/Under Ed. Perelman MI and Levashev YuN. "Moscow: Medicine and Life (2002).

3. Korzh AA., et al. "Operational access to the thoracic and lumbar vertebrae". Moscow: Medicine (1968). 
4. Root PG. "Surgery of bone and joint tuberculosis: in 3 tons". Leningrad: Medicine (1971).

5. "Bone and joint tuberculosis". Ed. Levashev, Yu N., Garbuza, A.E. Moscow: Medicine and Life (2003).

6. Perelman MI. "Physiatrics: National Leadership". Moscow: GEOTAR-Media (2007).

7. Ulrich EV and Mushkin AYu. "Vertebrology in terms, numbers, drawings". St. Petersburg: ELBI- St. Petersburg (2004).

8. Colmenero JD., et al. "Establishing the diagnosis of tuberculous vertebral osteomyelitis”. European Spine Journal 22.4 (2013): 579-586.

9. Garg RK and Somvanshi DS. "Spinal tuberculosis: a review". Journal of Spinal Cord Medicine 34.5 (2011): 440-454.

\section{Assets from publication with us}

- Prompt Acknowledgement after receiving the article

- Thorough Double blinded peer review

- Rapid Publication

- Issue of Publication Certificate

- High visibility of your Published work

Website: www.actascientific.com/

Submit Article: www.actascientific.com/submission.php

Email us: editor@actascientific.com

Contact us: +919182824667 